\title{
Gravitational effects on the neutrino oscillation
}

\author{
N. Fornengo, ${ }^{1,2, *}$ C. Giunti, ${ }^{2,3, \dagger}$ C. W. Kim, ${ }^{1, \$}$ and J. Song ${ }^{1, \S}$ \\ ${ }^{1}$ Department of Physics and Astronomy, The Johns Hopkins University, Baltimore, Maryland 21218 \\ ${ }^{2}$ INFN, Sezione di Torino, via P. Giuria 1, 10125 Torino, Italy \\ ${ }^{3}$ Dipartimento di Fisica Teorica, Università di Torino, via P. Giuria 1, 10125 Torino, Italy
}

(Received 12 November 1996)

\begin{abstract}
The propagation of neutrinos in a gravitational field is studied. A method of calculating a covariant quantum-mechanical phase in a curved space-time is presented. The result is used to calculate gravitational effects on the neutrino oscillation in the presence of a gravitational field. We restrict our discussion to the case of the Schwarzschild metric. Specifically, the cases of the radial propagation and the nonradial propagation are considered. A possible application to gravitational lensing of neutrinos is also suggested. [S0556-2821(97)04314-2]

PACS number(s): 95.85.Ry, 12.15.Ff, 14.60.Lm
\end{abstract}

\section{INTRODUCTION}

Neutrino oscillations in a flat space-time have been extensively studied in the past by using both plane waves [1] and wave packets [2] to represent the emitted neutrinos. In particular, it has been shown [2] that the standard treatment of neutrino oscillations in the plane wave approximation is valid only for extremely relativistic neutrinos, whereas for a general case, the wave packet treatment is essential. In this paper, we discuss how the results in a flat space-time are modified in a curved space-time. That is, we calculate the quantum-mechanical phase of neutrinos that are produced and propagate in a gravitational field. Our derivation of the neutrino oscillation formula in a gravitational field will be based on the covariant form of the quantum phase that arises due to the assumed mixing of massive neutrinos [3]. First we consider the case of neutrinos that are emitted and propagate in a radial trajectory in the Schwarzschild metric. Such a gravitational effect can, in principle, modify the standard vacuum oscillation formula for the solar and supernova neutrinos. Although the size of the effect is far beyond the current experimental detectability, in particular for the solar neutrinos, it may certainly be of interest for the neutrinos from very massive sources. (It is well known that the gravitational influence on the MSW effect for the solar neutrinos is significant if the equivalence principle is violated [4].) In our derivation we have not assumed a weak field approximation. We then compare our results with the previous results in the case of the radial trajectory obtained by [5-7] with clarifying remarks on the differences in the results and interpretations. As a further application, we also consider the case of nonradial propagation in a gravitational field. Finally, we discuss possible gravitational lensing effects on the neutrino oscillations, for which it is necessary to resort to the weak field approximation. It is to be noted that in the last two cases, due to the angular spread of neutrinos with different

\footnotetext{
*Electronic address: fornengo@jhup.pha.jhu.edu

${ }^{\dagger}$ Electronic address: giunti@to.infn.it

‡Electronic address: kim@eta.pha.jhu.edu

${ }^{\S}$ Electronic address: jhsong@eta.pha.jhu.edu
}

masses in the presence of the gravitational field, a proper way to treat the neutrinos is to resort to the wave packet formalism. (However, a complete, covariant description of neutrino propagation in a gravitational field in terms of wave packets is beyond the scope of this paper. This issue will be addressed elsewhere [8].) In order to discuss the problem in a transparent way, and in order to compare with the previous analyses, we restrict ourselves to the discussion of relativistic neutrinos, where a plane wave analysis can be employed.

The plan of the paper is as follows. In Sec. II, we briefly review the standard treatment of neutrino oscillations in a flat space-time using the plane wave formalism. In Sec. III we extend the plane wave analysis to the case in the presence of a gravitational field. For definiteness, we discuss the neutrino oscillations in a field described by the Schwarzschild metric. Specifically the cases of radial and nonradial propagation of neutrinos are discussed. In the last part of Sec. III, we suggest the possibility of gravitational lensing of neutrinos and evaluate the resulting flavor-changing oscillation probability.

\section{NEUTRINO PROPAGATION IN A FLAT SPACE-TIME}

Let us consider a neutrino produced at a space-time point $A\left(t_{A}, \vec{x}_{A}\right)$. Since it is produced by a weak interaction process, it emerges as a flavor eigenstate $\left|\nu_{\alpha}\right\rangle$, which is a superposition of the mass eigenstates $\left|\nu_{k}\right\rangle$, i.e.,

$$
\left|\nu_{\alpha}\right\rangle=\sum_{k} U_{\alpha k}^{*}\left|\nu_{k}\right\rangle
$$

where $U$ is the unitary mixing matrix of the neutrino fields. What actually propagates are the mass eigenstates, whose energy and momentum are $E_{k}$ and $\vec{p}_{k}$, respectively, and they are related by the mass-shell condition as

$$
E_{k}^{2}=\vec{p}_{k}^{2}+m_{k}^{2}
$$

Both $E_{k}$ and $\vec{p}_{k}$ are determined by the energy and momentum conservation at the production point $A$ and, in general, they 
are different for different mass eigenstates. In a flat spacetime, the propagation of the state $\left|\nu_{k}\right\rangle$ is described by a plane wave

$$
\left|\nu_{k}(t, \vec{x})\right\rangle=\exp \left(-i \Phi_{k}\right)\left|\nu_{k}\right\rangle
$$

where

$$
\Phi_{k}=E_{k} t-\vec{p}_{k} \cdot \vec{x} .
$$

Neutrino oscillations take place due to the fact that different states $\left|\nu_{k}\right\rangle$ propagate differently because they have different energies, momenta, and masses. When they arrive at a detector located at a space-time point $B\left(t_{B}, \vec{x}_{B}\right)$ which detects flavor eigenstates via a weak interaction process, they have developed a relative shift in their phases. In order for the oscillation to occur and to be observed, some requirements must be met. First, in addition to the standard assumption of mixing of massive neutrinos, the mass eigenstates must be produced coherently. This implies that interference is possible only among mass eigenstates produced in the same process, because neutrinos produced by different processes have, in general, random relative phases in their wave functions, which destroy the coherence. Second, the states have to be detected at the same time $t_{B}$ and at the same place $\vec{x}_{B}$.

Under these circumstances, the interference can take place and the oscillation phenomenon arises. The probability that the neutrino produced as $\left|\nu_{e}\right\rangle$ is detected as $\left|\nu_{\mu}\right\rangle$ is, therefore (in the case of two generations, where $U$ is parametrized as a function of the mixing angle $\theta$ in the usual way) [9]

$$
\mathcal{P}\left(\nu_{e} \rightarrow \nu_{\mu}\right)=\left|\left\langle\nu_{\mu} \mid \nu_{e}\left(t_{B}, \vec{x}_{B}\right)\right\rangle\right|^{2}=\sin ^{2}(2 \theta) \sin ^{2}\left(\frac{\Phi_{12}}{2}\right),
$$

where $\Phi_{12}=\Phi_{1}-\Phi_{2}$ and $\Phi_{k}(k=1,2)$ are the phases,

$$
\Phi_{k}=E_{k}\left(t_{B}-t_{A}\right)-\vec{p}_{k} \cdot\left(\vec{x}_{B}-\vec{x}_{A}\right)=E_{k} \int_{t_{A}}^{t_{B}} d t-\vec{p}_{k} \cdot \int_{\vec{x}_{A}}^{\vec{x}_{B}} d \vec{x},
$$

acquired by the mass eigenstates.

The expression for the phase $\Phi_{k}$ in Eq. (6) can be written in a covariant form, which is suitable for the subsequent application in a curved space-time, as [10]

$$
\Phi_{k}=\int_{A}^{B} p_{\mu}^{(k)} d x^{\mu}
$$

where

$$
p_{\mu}^{(k)}=m_{k} g_{\mu \nu} \frac{d x^{\nu}}{d s}
$$

is the canonical conjugate momentum to the coordinates $x^{\mu}$ and $g_{\mu \nu}$ and $d s$ are the metric tensor and the line element, respectively. This covariant phase in Eq. (7) was first discussed by Stodolsky [10], and has been used in [5-7] to calculate the neutrino oscillation phase difference.

Equation (5) represents the oscillation probability for a neutrino produced at the space-time point $A\left(t_{A}, \vec{x}_{A}\right)$ and de- tected at a given space-time position $B\left(t_{B}, \vec{x}_{B}\right)$. In actual experiments, however, the time difference $\left(t_{B}-t_{A}\right)$ is not measured, whereas the relative position $\left|\vec{x}_{B}-\vec{x}_{A}\right|$ of the source and the detector is known. In the plane wave formalism, this can be taken care of consistently only for relativistic neutrinos by replacing $\left(t_{B}-t_{A}\right)$ with [11]

$$
\left(t_{B}-t_{A}\right) \simeq\left|\vec{x}_{B}-\vec{x}_{A}\right|
$$

and thus the time difference does not appear in the formula for the oscillation probability. In this approximation, the phase of Eq. (6) becomes

$$
\Phi_{k}=\left(E_{k}-\left|\vec{p}_{k}\right|\right)\left|\vec{x}_{B}-\vec{x}_{A}\right| .
$$

Applying the relativistic expansion $m_{k} \ll E_{k}$, we can approximate, to the first order,

$$
E_{k} \simeq E_{0}+O\left(\frac{m_{k}^{2}}{2 E_{0}}\right)
$$

where $E_{0}$ is the energy for a massless neutrino. Therefore, we have

$$
E_{k}-\left|\vec{p}_{k}\right|=E_{k}-\sqrt{E_{k}^{2}-m_{k}^{2}} \simeq \frac{m_{k}^{2}}{2 E_{0}},
$$

which leads to the standard result for the phase:

$$
\Phi_{k} \simeq \frac{m_{k}^{2}}{2 E_{0}}\left|\vec{x}_{B}-\vec{x}_{A}\right| .
$$

The phase difference responsible for the oscillation can be given by Eq. (13) as

$$
\Phi_{k j} \simeq \frac{\Delta m_{k j}^{2}}{2 E_{0}}\left|\vec{x}_{B}-\vec{x}_{A}\right|,
$$

where $\Delta m_{k j}^{2}=m_{k}^{2}-m_{j}^{2}$.

For more general situations, where some or all of the states $\nu_{k}$ are nonrelativistic, the above discussion cannot be applied, and a wave packet analysis is required [2]. In this case, the relation in Eq. (9) is no longer valid, and moreover the problem of the coherence of the different states at the detection position has to be taken into account. However, for relativistic neutrinos, the wave packet formalism shows that the approximation of Eq. (9) is indeed appropriate, and the oscillation probability $\mathcal{P}\left(\nu_{e} \rightarrow \nu_{\mu}\right)$ has the form of Eq. (5), where the phase shift $\Phi_{k j}$ is given by Eq. (14).

\section{NEUTRINO PROPAGATION IN THE SCHWARZSCHILD METRIC}

Let us now turn to the discussion of the propagation of neutrinos in a gravitational field. For the sake of definiteness, and also because it may represent a situation of possible physical interest, we will discuss the propagation in a gravitational field of a nonrotating spherically symmetric object, which is described by the Schwarzschild metric. The situation under consideration can be described by the line element in the coordinate frame $\{t, r, \vartheta, \phi\}$ as 


$$
d s^{2}=B(r) d t^{2}-B(r)^{-1} d r^{2}-r^{2} d \vartheta^{2}-r^{2} \sin ^{2} \vartheta d \phi^{2},
$$

where

$$
B(r)=\left(1-\frac{2 G M}{r}\right)
$$

$G$ is the Newtonian constant and $M$ denotes the mass of the source of the gravitational field. Since the gravitational field is isotropic, the classical orbit may be confined to a plane. Hence, we can choose it to be on the equatorial plane $\vartheta=\pi / 2$, and we have $d \vartheta=0$.

The relevant components of the canonical momentum $p_{\mu}^{(k)}$ of Eq. (8) are

$$
\begin{gathered}
p_{t}^{(k)}=m_{k} B(r) \frac{d t}{d s}, \\
p_{r}^{(k)}=-m_{k} B(r)^{-1} \frac{d r}{d s}, \\
p_{\phi}^{(k)}=-m_{k} r^{2} \frac{d \phi}{d s},
\end{gathered}
$$

and they are related to each other and to the mass $m_{k}$ by the mass-shell relation

$$
m_{k}^{2}=g^{\mu \nu} p_{\mu}^{(k)} p_{\nu}^{(k)}=\frac{1}{B(r)}\left(p_{t}^{(k)}\right)^{2}-B(r)\left(p_{r}^{(k)}\right)^{2}-\frac{\left(p_{\phi}^{(k)}\right)^{2}}{r^{2}} .
$$

The fact that the metric tensor components do not depend on the coordinates $t$ and $\phi$ ensures that their canonical momenta $p_{t}^{(k)}$ and $p_{\phi}^{(k)}$ are constant along the trajectory. We define the constant of motion to be $E_{k} \equiv p_{t}^{(k)}$ and $J_{k} \equiv-p_{\phi}^{(k)}$. They represent the energy and the angular momentum which an observer, located at $r=\infty$, sees for the mass eigenstate $\nu_{k}$. They differ from the energy and the angular momentum measured by an observer at a position $r_{B}$ or those at production point $r_{A}$. The correct way to define the energies which are actually involved in a realistic situation is not, in general, unique. For example, for a neutrino produced in the almost stationary shock wave of a supernova, a local static reference frame for the production point $r_{A}$ seems appropriate. On the contrary, for neutrinos produced in the accretion disk around a black hole, a free-falling orbiting system seems proper. Similar arguments apply to the detection of neutrinos. For example, in the case of solar neutrinos, the detectors are in the free-falling frame. The general situation can be rather complicated and every case must be carefully dealt with. In our discussion we will choose the local reference frame. The local energy, defined as the energy measured by an observer at rest at a position $r$, is related to $E_{k}$ from the transformation law which relates the local reference frame $\left\{x^{\hat{\alpha}}\right\}=\{\hat{t}, \hat{r}, \hat{\varphi}, \hat{\theta}\}$ to the frame $\left\{x^{\mu}\right\}=\{t, r, \varphi, \theta\}[12]$,

$$
x^{\hat{\alpha}}=L_{\mu}^{\hat{\alpha}} x^{\mu}, \quad g_{\mu \nu}=L^{\hat{\alpha}}{ }_{\mu} L_{\nu}^{\hat{\beta}} \eta_{\hat{\alpha} \hat{\beta}},
$$

where $L^{\hat{\alpha}}{ }_{\mu}$ are the coefficients of the transformation between the two bases:

$$
\begin{gathered}
L_{t}^{\hat{t}}=\sqrt{\left|g_{t t}\right|}, \quad L_{r}^{\hat{r}_{r}}=\sqrt{\left|g_{r r}\right|}, \quad L_{\vartheta}^{\hat{\vartheta}}=\sqrt{\left|g_{\vartheta \vartheta}\right|}, \quad L_{\varphi}^{\hat{\varphi}}=\sqrt{\left|g_{\varphi \varphi}\right|}, \\
\text { others }=0 .
\end{gathered}
$$

Therefore, the local energy is

$$
E_{k}^{(\text {loc })}(r)=\left|g_{t t}\right|^{-1 / 2} E_{k}=B(r)^{-1 / 2} E_{k} .
$$

In order to obtain the neutrino oscillation probability in a gravitational field, we will calculate the interference of the wave functions of different mass eigenstates created at a space-time point $A$ and detected at a space-time point $B$. In the plane wave approximation, the phase of each mass eigenstate $\nu_{k}$ is defined by the covariant expression in Eq. (7) and the interference of the $k$ th and $j$ th mass eigenstates is given by the phase difference

$$
\Phi_{k j}=\int_{A}^{B}\left(p_{\mu}^{(k)}-p_{\mu}^{(j)}\right) d x^{\mu}=\Phi_{k}-\Phi_{j}
$$

Here the integration must be made on a definite space-time trajectory from $A$ to $B$. Following the standard treatment of the oscillations of the relativistic neutrinos in a flat spacetime, as discussed in Sec. II, we will calculate the interference phase in Eq. (24) along the light-ray trajectory from $A$ to $B$. This corresponds to the approximation in Eq. (9) for the flat space-time case. We emphasize that the phases in Eq. (24) are not the phases on the classical trajectory of the mass eigenstates [10] but the phases calculated on the light-ray trajectory. We will see that for relativistic neutrinos the result for the phase difference in Eq. (24) is proportional to $\Delta m_{k j}^{2} / 2 E_{0}$, as in the standard treatment of neutrino oscillations in a flat space-time.

We will now define the phase acquired by the mass eigenstate $\nu_{k}$ when it travels from point $A\left(t_{A}, r_{A}, \phi_{A}\right)$ to point $B\left(t_{B}, r_{B}, \phi_{B}\right)$ as

$$
\Phi_{k}=\int_{A}^{B}\left[E_{k} d t-p_{k}(r) d r-J_{k} d \phi\right],
$$

where we have defined $p_{k}(r) \equiv-p_{r}^{(k)}$. The integration in Eq. (25) is performed along the light-ray trajectory which links the space-time points $A$ and $B$. At this stage, we note that $E_{k}$ and $J_{k}$, which are constants of motion for the geodesic trajectory of the $k$ th eigenstate, are no longer constant along the light-ray trajectory. Instead, the energy at infinity $E_{0}$ and the angular momentum $J_{0}$ at infinity for a massless particle are constant along the light-ray path. Therefore, $E_{k}$ and $J_{k}$ cannot be taken out of the integration in Eq. (25) and some caution is required for the calculation. We will show explicitly in the following subsections, however, that in the relativistic limit, this problem can be circumvented.

We now discuss two different situations: radial propagation and nonradial propagation. In the last subsection we will address the possibility of gravitational lensing. 


\section{A. Radial propagation}

For neutrinos propagating in a radial direction, we have $d \phi=0$ and no angular momentum. Equation (25) is reduced to

$$
\Phi_{k}=\int_{r_{A}}^{r_{B}}\left[E_{k}\left(\frac{d t}{d r}\right)_{0}-p_{k}(r)\right] d r
$$

where $p_{k}(r)$ is obtained, from the mass-shell relation, Eq. (20), with $J_{k}=0$, as

$$
p_{k}(r)= \pm \frac{1}{B(r)} \sqrt{E_{k}^{2}-B(r) m_{k}^{2}}
$$

and the light-ray differential $(d t / d r)_{0}$ is

$$
\left(\frac{d t}{d r}\right)_{0}= \pm \frac{1}{B(r)}
$$

In Eqs. (27) and (28), the sign ( \pm ) applies to neutrinos propagating outward $(+)$ or inward $(-)$ of the gravitational well, respectively. Therefore, the quantum-mechanical phase $\Phi_{k}$ is

$$
\Phi_{k}= \pm \int_{r_{A}}^{r_{B}}\left[E_{k}-\sqrt{E_{k}^{2}-B(r) m_{k}^{2}}\right] \frac{d r}{B(r)} .
$$

At this point, we apply the relativistic expansion using the energy at infinity $E_{k}$ as a reference value, i.e., $m_{k} \ll E_{k}$. As in the flat space-time case, the following relation holds:

$$
E_{k} \simeq E_{0}+O\left(\frac{m_{k}^{2}}{2 E_{0}}\right),
$$

where $E_{0}$ is the energy at infinity for a massless particle. Taking into account that $0<B(r) \leqslant 1$, we have

$$
\sqrt{E_{k}^{2}-B(r) m_{k}^{2}} \simeq E_{k}-B(r) \frac{m_{k}^{2}}{2 E_{0}} .
$$

Then, the phase in Eq. (29) is approximated by

$$
\Phi_{k} \simeq \pm \int_{r_{A}}^{r_{B}} \frac{m_{k}^{2}}{2 E_{0}} d r .
$$

Since the integration is performed along the light-ray trajectory, $E_{0}$ is constant and the integration is easily performed to give

$$
\Phi_{k} \simeq \frac{m_{k}^{2}}{2 E_{0}}\left|r_{B}-r_{A}\right|
$$

The phase shift which determines the oscillation is, therefore,

$$
\Phi_{k j} \simeq \frac{\Delta m_{k j}^{2}}{2 E_{0}}\left|r_{B}-r_{A}\right|
$$

We note that the derivation of this result does not depend on the weak field approximation.
The result for the phase shift in Eq. (34) is in agreement with that in Ref. [7], but it has been obtained with a different approach. The authors of Ref. [7] calculated the phase of each massive neutrino along its classical trajectory. The classical trajectories of different massive neutrinos reaching the detection point at the same time must start from the production point at different times. Hence, there is an initial phase difference among the wave functions of different massive neutrinos which must be added "by hand." Instead, our approach has been to calculate the interference between mass eigenstates produced at the same space-time position and detected at the same space-time point, related by the light-ray relation of Eq. (28). We think that our approach is natural for the calculation of interference effects of particles with slightly different masses and can be considered as a natural extension of the usual approach for the calculation of neutrino and kaon oscillations in flat space-time. On the other hand, any comparison of our result with that in Ref. [5] is problematic since the energy $E$ used in Ref. [5] is not clearly defined.

Some comments on the definition of "relativistic" neutrinos are in order here. Let us consider the following cases:

(1) $m_{k}^{2} \ll E_{k}^{2}$ (relativistic at infinity),

(2) $m_{k}^{2} \ll\left[E_{k}^{(\text {loc })}\left(r_{A}\right)\right]^{2}$ (relativistic at the source),

(3) $m_{k}^{2} \ll\left[E_{k}^{(\text {loc })}\left(r_{B}\right)\right]^{2}$ (relativistic at the detector).

In case (1), the ratio of $m_{k}^{2}$ to any local energy $E_{k}^{(\text {loc })}(r)$ is, from Eq. (23) and $B(r) \leqslant 1$,

$$
\frac{m_{k}^{2}}{\left[E_{k}^{(\text {loc })}(r)\right]^{2}}=\frac{m_{k}^{2}}{E_{k}^{2}} B(r) \leqslant \frac{m_{k}^{2}}{E_{k}^{2}} \ll 1,
$$

so that the neutrinos are even more relativistic at $r<\infty$, and the approximation in Eq. (31) is certainly justified.

Case (2) needs a caution when the observer happens to be at infinity, because the ratio of $m_{k}^{2}$ to the energy at $r=\infty$ becomes

$$
\frac{m_{k}^{2}}{E_{k}^{2}}=\frac{m_{k}^{2}}{\left[E_{k}^{(\text {loc })}\left(r_{A}\right)\right]^{2}} \frac{1}{B\left(r_{A}\right)} .
$$

That is, even if neutrinos are produced highly relativistically, they are not guaranteed to be relativistic at $r=\infty$, unless $r_{A} \gg 2 G M\left[1-\left(m_{k} / E_{k}^{(\text {loc })}\left(r_{A}\right)\right)^{2}\right]^{-1}$. For the obvious reason that nonrelativistic neutrinos cannot be detected, at least with known techniques (assuming that neutrino masses are much smaller than $1 \mathrm{MeV}$ ), however, the lack of validity of the relativistic condition at infinity not only means that the approximate formula (31) is not valid, but also that in practice such neutrinos are not detectable at infinity.

Case (3) deals with an observer under the influence of a sizable gravitational field. In this case, neutrinos stay always relativistic along their path $\left(r_{A}<r<r_{B}\right)$, which validates approximation in Eq. (31), for we have

$$
B(r) \frac{m_{k}^{2}}{E_{k}^{2}}=\frac{B(r)}{B\left(r_{B}\right)} \frac{m_{k}^{2}}{\left[E_{k}^{(\text {loc }}\left(r_{B}\right)\right]^{2}} \leqslant \frac{m_{k}^{2}}{\left[E_{k}^{(\text {loc })}\left(r_{B}\right)\right]^{2}} \ll 1 .
$$


In short, neutrinos are assumed to be "relativistic" when they are relativistic at infinity, relativistic at the detector, or relativistic at the production point with $r_{A} \gg 2 G M\left\{1-\left[m_{k} / E_{k}^{(\mathrm{loc})}\left(r_{A}\right)\right]^{2}\right\}^{-1}$ and then Eq. (33) provides the correct quantum phase.

As a final comment, we wish to compare Eq. (33) with that of the flat space-time case. As they stand, the expressions of the phase in Eq. (33) and the phase shift in Eq. (34) appear identical to those of the flat space-time case. However, the gravitational effects are present implicitly in Eqs. (33) and (34). In the absence of a gravitational field, $E_{0}$ is the energy of the neutrino as seen by any observer along its trajectory, and $\left(r_{B}-r_{A}\right)$ is the distance over which a neutrino propagates. Therefore, Eq. (33) gives the standard result shown in Eq. (13). However, in the presence of gravity, the propagation of a neutrino is over its proper distance

$$
\begin{aligned}
L_{p} \equiv & \int_{r_{A}}^{r_{B}} \sqrt{g_{r r}} d r \\
= & r_{B} \sqrt{1-\frac{2 G M}{r_{B}}}-r_{A} \sqrt{1-\frac{2 G M}{r_{A}}} \\
& +2 G M\left[\ln \left(\sqrt{r_{B}-2 G M}+\sqrt{r_{B}}\right)\right. \\
& \left.-\ln \left(\sqrt{r_{A}-2 G M}+\sqrt{r_{A}}\right)\right] .
\end{aligned}
$$

To simplify the following discussion, we consider the case of a weak field, where $L_{p}$ is approximated to

$$
L_{p} \simeq r_{B}-r_{A}+G M \ln \frac{r_{B}}{r_{A}} .
$$

This shows that, in a gravitational field, the effective length in the phase [i.e., $\left.\left(r_{B}-r_{A}\right)\right]$ is shorter than $L_{p}$. Moreover, the energy measured by a detector at $r_{B}$ is not $E_{0}$, but rather the local value $E_{0}{ }^{\left({ }^{l o c}\right)}\left(r_{B}\right)$. When expressed in terms of the local energy and the proper distance, the phase shift $\Phi_{k j}$ of Eq. (34) in the weak field approximation is

$$
\Phi_{k j} \simeq\left(\frac{\Delta m_{k j}^{2} L_{p}}{2 E_{0}{ }^{(\mathrm{loc})}\left(r_{B}\right)}\right)\left[1-G M\left(\frac{1}{L_{p}} \ln \frac{r_{B}}{r_{A}}-\frac{1}{r_{B}}\right)\right] .
$$

The first parenthesis on the right-hand side in Eq. (40) is analogous to the flat space-time oscillation phase. The second square parenthesis represents the correction due to the gravitational effects.

The proper oscillation length $L_{k j}^{\text {osc }}$, in the weak field approximation, is

$$
\begin{aligned}
L_{k j}^{\mathrm{osc}}\left(r_{B}\right)= & \frac{4 \pi E_{0}^{\mathrm{loc}}\left(r_{B}\right)}{\Delta m_{k j}^{2}}-G M\left[\ln \left(1-\frac{4 \pi E_{0}{ }^{\mathrm{loc}}\left(r_{B}\right)}{\Delta m^{2} r_{B}}\right)\right. \\
& \left.+\frac{4 \pi E_{0}^{\mathrm{loc}}\left(r_{B}\right)}{\Delta m^{2} r_{B}}\right]
\end{aligned}
$$

where the quantity in square brackets is negative. We conclude, therefore, that the proper oscillation length is increased in the gravitational field, as expected.

\section{B. Nonradial propagation}

In this subsection, we discuss the case of the propagation along a general trajectory. In contrast to the radial case, the motion has an additional angular dependence. The phase $\Phi_{k}$ is

$$
\Phi_{k}=\int_{r_{A}}^{r_{B}}\left[E_{k}\left(\frac{d t}{d r}\right)_{0}-p_{k}(r)-J_{k}\left(\frac{d \phi}{d r}\right)_{0}\right] d r,
$$

where the integral is taken along the light-ray trajectory that links the production point $A$ to the detection point $B$. In Eq. (42), the quantities $(d t / d r)_{0}$ and $(d \phi / d r)_{0}$ along the lightray trajectory are

$$
\begin{gathered}
\left(\frac{d t}{d r}\right)_{0}=\frac{E_{0}}{B^{2}(r) p_{0}(r)}, \\
\left(\frac{d \phi}{d r}\right)_{0}=\frac{J_{0}}{r^{2}} \frac{1}{B(r) p_{0}(r)} .
\end{gathered}
$$

It is convenient to express the angular momentum $J_{k}$ as a function of the energy $E_{k}$, the impact parameter $b$, and the velocity at infinity $v_{k}^{(\infty)}[13]$ :

$$
J_{k}=E_{k} b v_{k}^{(\infty)} \text {. }
$$

Since at $r=\infty$ the metric is Minkowskian (no gravity), we can write

$$
v_{k}^{(\infty)}=\frac{\sqrt{E_{k}^{2}-m_{k}^{2}}}{E_{k}} \simeq 1-\frac{m_{k}^{2}}{2 E_{k}^{2}},
$$

where in the last equality we used the relativistic approximation up to the order $O\left(m_{k}^{2} / E_{k}^{2}\right)$. The angular momentum of a massless particle, $J_{0}$, is obviously

$$
J_{0}=E_{0} b .
$$

With Eqs. (43)-(46), the expression of $\Phi_{k}$ in Eq. (42) can be conveniently arranged as

$$
\begin{aligned}
\Phi_{k}= & \int_{r_{A}}^{r_{B}} d r \frac{E_{0}}{B(r) p_{0}(r)}\left[\frac{E_{k}}{B(r)}-\frac{B(r) p_{0}(r)}{E_{0}} p_{k}(r)\right. \\
& \left.-\frac{E_{k} b^{2}}{r^{2}}\left(1-\frac{m_{k}^{2}}{2 E_{k}^{2}}\right)\right] .
\end{aligned}
$$

The mass-shell condition, Eq. (20), gives

$$
\begin{gathered}
B(r) p_{0}(r)= \pm E_{0} \sqrt{1-B(r) \frac{b^{2}}{r^{2}}}, \\
B(r) p_{k}(r)= \pm E_{k} \sqrt{1-B(r) \frac{b^{2}}{r^{2}}-B(r) \frac{m_{k}^{2}}{E_{k}^{2}}\left(1-\frac{b^{2}}{r^{2}}\right)} \\
\simeq \pm E_{k} \sqrt{1-B(r) \frac{b^{2}}{r^{2}}}\left[1-\frac{B(r)\left(1-b^{2} / r^{2}\right)}{1-B(r) b^{2} / r^{2}} \frac{m_{k}^{2}}{2 E_{k}^{2}}\right] .
\end{gathered}
$$


The last approximate equality in Eq. (49) is due to the relativistic expansion. In Eqs. (48) and (49), the sign ( \pm ) is determined by whether $d r$ is positive $(+)$ or negative $(-)$. Substitution of Eqs. (48) and (49) into Eq. (47) simplifies the expression for the phase $\Phi_{k}$ to

$$
\Phi_{k} \simeq \int_{r_{A}}^{r_{B}} d r \frac{E_{0}}{B(r) p_{0}(r)} E_{k} \frac{m_{k}^{2}}{2 E_{k}^{2}} .
$$

Since the following relation holds, in the relativistic approximation of Eq. (30),

$$
E_{k} \frac{m_{k}^{2}}{2 E_{k}^{2}} \simeq E_{0} \frac{m_{k}^{2}}{2 E_{0}^{2}},
$$

$\Phi_{k}$ can be expressed as

$$
\Phi_{k} \simeq \pm \frac{m_{k}^{2}}{2 E_{0}} \int_{r_{A}}^{r_{B}} \frac{d r}{\sqrt{1-B(r)\left(b^{2} / r^{2}\right)}} .
$$

Equation (52) is the phase acquired by the mass eigenstate $\left|\nu_{k}\right\rangle$ for a nonradial propagation from the source $A$ to the detector $B$. In the limit $b \rightarrow 0$, which reduces the motion to be radial, Eq. (33) is recovered. We also notice that the integrand in Eq. (52) is divergent at the point of the closest approach $r_{0}$, defined by the condition that the rate of change of the coordinate $r$ with respect to the angle $\phi$ vanishes:

$$
\frac{d r}{d \phi}=0 \Rightarrow E_{0}^{2}=\frac{J_{0}^{2}}{r_{0}^{2}} B\left(r_{0}\right) \Rightarrow 1-\frac{b^{2}}{r_{0}^{2}} B\left(r_{0}\right)=0 .
$$

However, the integral which gives the phase $\Phi_{k}$ is finite. We will show this explicitly in the weak field approximation.

The expression of $\Phi_{k}$ obtained in Eq. (52) is valid for any spherically symmetric (and time-independent) field. It has been derived without any assumption on the strength of the gravitational field. In order to gain more physical insight, however, we perform a weak field approximation, which allows us to perform the integration analytically. The approximation is valid if the field is weak enough to satisfy the condition $G M \ll r$ for all the $r$ 's along the trajectory under consideration. For example, the gravitational field of the sun at its surface is about $G M_{\odot} / R_{\odot} \sim 2 \times 10^{-6}$ and that of a galaxy is about $G\left(10^{11} M_{\odot}\right) / 30 \mathrm{kpc} \sim 1.6 \times 10^{-7}$, both of which justify the weak field approximation. Whenever the weak field approximation is applied, we keep the expansion up to the order $O(G M / r)$.

First, let us consider the case where a neutrino is produced in a gravitational field and then propagates outward from the potential well nonradially. The weak field approximation allows us to expand

$$
\sqrt{1-B(r) \frac{b^{2}}{r^{2}}} \simeq \sqrt{1-\frac{b^{2}}{r^{2}}}\left[1+\frac{G M}{r} \frac{b^{2}}{r^{2}-b^{2}}\right] .
$$

The phase $\Phi_{k}$ is then easily integrated and becomes

$$
\Phi_{k} \simeq \frac{m_{k}^{2}}{2 E_{0}}\left[\sqrt{r_{B}^{2}-b^{2}}-\sqrt{r_{A}^{2}-b^{2}}\right.
$$

$$
\left.+G M\left(\frac{r_{B}}{\sqrt{r_{B}^{2}-b^{2}}}-\frac{r_{A}}{\sqrt{r_{A}^{2}-b^{2}}}\right)\right] .
$$

We notice again, as a consistency check, that the radial limit $b \rightarrow 0$ gives the same expression as given in Eq. (33).

The second situation is when a neutrino moves around the massive object, crossing the closest approach point at $r=r_{0}$. Taking into account the sign of the momentum, the phase is

$$
\begin{aligned}
\Phi_{k}\left(r_{A} \rightarrow r_{0} \rightarrow r_{B}\right)= & \frac{m_{k}^{2}}{2 E_{0}} \int_{r_{0}}^{r_{A}} \frac{d r}{\sqrt{1-B(r)\left(b^{2} / r^{2}\right)}} \\
& +\frac{m_{k}^{2}}{2 E_{0}} \int_{r_{0}}^{r_{B}} \frac{d r}{\sqrt{1-B(r)\left(b^{2} / r^{2}\right)}} .
\end{aligned}
$$

The position of the closest approach can be solved from Eq. (53) in the weak field approximation, as

$$
r_{0}=b\left(1-\frac{G M}{b}\right) .
$$

Substituting Eq. (57) into Eq. (56), we have

$$
\begin{aligned}
\Phi_{k} \simeq & \frac{m_{k}^{2}}{2 E_{0}}\left[\sqrt{r_{A}^{2}-r_{0}^{2}}+\sqrt{r_{B}^{2}-r_{0}^{2}}+G M\left(\sqrt{\frac{r_{A}-r_{0}}{r_{A}+r_{0}}}\right.\right. \\
& \left.\left.+\sqrt{\frac{r_{B}-r_{0}}{r_{B}+r_{0}}}\right)\right] \\
\simeq & \frac{m_{k}^{2}}{2 E_{0}}\left[\sqrt{r_{A}^{2}-b^{2}}+\sqrt{r_{B}^{2}-b^{2}}\right. \\
& +G M\left(\frac{b}{\sqrt{r_{A}^{2}-b^{2}}}+\frac{b}{\sqrt{r_{B}^{2}-b^{2}}}+\sqrt{\frac{r_{A}-b}{r_{A}+b}}\right. \\
& \left.\left.+\sqrt{\frac{r_{B}-b}{r_{B}+b}}\right)\right] .
\end{aligned}
$$

We observe that, in this case, the radial limit $b \rightarrow 0$ is meaningless, because it would correspond to a radial motion which crosses the gravitational source, where our description becomes inadequate.

For $b \ll r_{A, B}$, Eq. (58) is reduced to [up to the order of $\left.\left(b^{2} / r_{A, B}^{2}\right)\right]$

$$
\Phi_{k}=\frac{m_{k}^{2}}{2 E_{0}}\left(r_{A}+r_{B}\right)\left[1-\frac{b^{2}}{2 r_{A} r_{B}}+\frac{2 G M}{r_{A}+r_{B}}\right],
$$

which will be used to discuss the gravitational lensing in the following subsection. It is interesting to note that Eq. (59) has a gravitational effect which does not depend on the distance between the source and the detector (assuming that this distance is much larger than the impact parameter $b$ ). That is, this gravitational effect integrated along a trajectory which passes close to a gravitational center induces a constant phase shift $2 G M\left(m_{k}^{2} / 2 E_{0}\right)$. Furthermore, this constant phase shift does not depend on how close the trajectory passes to the gravitational center. Therefore, if, for example, 
a neutrino travels relatively close to several well-separated gravitational centers, the net phase shift becomes the sum of the phase shifts induced by each gravitational center.

\section{Gravitational lensing of neutrinos}

Let us consider a gravitational lens which is located between a source and an observer but off the line connecting the two. A neutrino emitted from the source can travel along two different paths, the proper distances of which are different and give the quantum interference at the detector.

Oscillations arise due to the interference not only between the mass eigenstates $\nu_{k}$ and $\nu_{j}$ traveling along each path, but also between the mass eigenstates propagating along different paths [for definiteness, we denote them as long path $(L)$ and short path $(S)]$. A neutrino produced as a flavor eigenstate $\left|\nu_{e}\right\rangle=\cos \theta\left|\nu_{1}\right\rangle+\sin \theta\left|\nu_{2}\right\rangle \quad$ at the source $A\left(t_{A}, r_{A}, \phi_{A}\right)$, evolves into (we consider only two generations)

$$
\begin{aligned}
\left|\nu_{e}, B\right\rangle= & N \sum_{\text {path }=L, S}\left[\cos \theta \exp \left(-i \Phi_{1}^{\text {path }}\right)\left|\nu_{1}\right\rangle\right. \\
& \left.+\sin \theta \exp \left(-i \Phi_{2}^{\text {path }}\right)\left|\nu_{2}\right\rangle\right],
\end{aligned}
$$

where $N$ is the normalization constant. The flavor-changing oscillation probability at the detector is then given by

$$
\begin{aligned}
\mathcal{P}\left(\nu_{e} \rightarrow \nu_{\mu}\right)= & \left|\left\langle\nu_{\mu} \mid \nu_{e}, B\right\rangle\right|^{2} \\
= & \frac{1}{2} \cos ^{2} \theta \sin ^{2} \theta\left[1+\cos \left(\Phi_{1}^{L}-\Phi_{1}^{S}\right)\right. \\
& +1+\cos \left(\Phi_{2}^{L}-\Phi_{2}^{S}\right)-\left\{\cos \left(\Phi_{2}^{L}-\Phi_{1}^{L}\right)\right. \\
& \left.+\cos \left(\Phi_{2}^{S}-\Phi_{1}^{S}\right)\right\}-\left\{\cos \left(\Phi_{2}^{L}-\Phi_{1}^{S}\right)\right. \\
& \left.\left.+\cos \left(\Phi_{2}^{S}-\Phi_{1}^{L}\right)\right\}\right] .
\end{aligned}
$$

The phases $\Phi_{k}^{\text {path }}$ in Eq. (61) can be evaluated along the light-ray trajectories as shown in the previous subsection. Substituting Eq. (59) into Eq. (61), we have

$$
\begin{aligned}
\mathcal{P}\left(\nu_{e} \rightarrow \nu_{\mu}\right)= & \sin ^{2}(2 \theta)\left[\sin ^{2}\left\{\frac{\Delta m^{2} X}{4 E_{0}}\left(1+\frac{2 G M}{X}-\frac{\Sigma b^{2}}{4 r_{A} r_{B}}\right)\right\}\right. \\
& \times \cos \left(\frac{m_{1}^{2} X}{4 E_{0}} \frac{\Delta b^{2}}{2 r_{A} r_{B}}\right) \cos \left(\frac{m_{2}^{2} X}{4 E_{0}} \frac{\Delta b^{2}}{2 r_{A} r_{B}}\right) \\
& \left.+\sin ^{2}\left(\frac{\Sigma m^{2} X}{4 E_{0}} \frac{\Delta b^{2}}{4 r_{A} r_{B}}\right) \sin ^{2}\left(\frac{\Delta m^{2} X}{4 E_{0}} \frac{\Delta b^{2}}{4 r_{A} r_{B}}\right)\right],
\end{aligned}
$$

where we have defined $X \equiv r_{A}+r_{B}, \quad \Delta m^{2} \equiv m_{2}^{2}-m_{1}^{2}$, $\Sigma m^{2} \equiv m_{2}^{2}+m_{1}^{2}, \Delta b^{2} \equiv b_{L}^{2}-b_{S}^{2}$, and $\Sigma b^{2} \equiv b_{L}^{2}+b_{S}^{2}$. In the symmetric case where the lens is aligned with the source and the detector, $\Delta b^{2}=0$ and $\Sigma b^{2}=2 b^{2}$ and the above flavorchanging probability is reduced to that of the nonradially propagating neutrinos,

$$
\begin{aligned}
\left.\mathcal{P}\left(\nu_{e} \rightarrow \nu_{\mu}\right)\right|_{\left(\Delta b^{2}=0\right)}= & \sin ^{2}(2 \theta) \sin ^{2}\left[\frac { \Delta m ^ { 2 } X } { 4 E _ { 0 } } \left(1+\frac{2 G M}{X}\right.\right. \\
& \left.\left.-\frac{b^{2}}{2 r_{A} r_{B}}\right)\right]
\end{aligned}
$$

which can be obtained directly from Eq. (59). This is expected, since the symmetric case is equivalent to the case of the nonradial propagation.

Obviously, the proper way to discuss the gravitational lensing effects on the neutrino oscillations would require a wave packet formalism. Such a study is beyond the scope of the present paper and will be given elsewhere.

\section{CONCLUSION}

We have studied the propagation of neutrinos in a curved space-time and the modification to the neutrino oscillation by calculating a covariant quantum-mechanical phase $\Phi_{k}$. The gravitational field considered in this work is that of a nonrotating spherically symmetric object, described by the Schwarzschild metric. Furthermore, we have assumed that neutrinos are relativistic so that a plane wave analysis can be applied.

Radial and nonradial propagation have been discussed in the light-ray approximation. Although our phase for the radial motion is in agreement with the result of the previous work [7], the interpretations are different. Any comparison of our result with that in [5] is problematic since the energy used in [5] is not clearly defined.

The calculated phase appears identical to that of the flat space-time case. This is because the phase is expressed in terms of the asymptotic energy $E_{0}$ and the coordinate distance. However, the gravitational effects do appear in the leading order if we express the phase with the locally measured energy and proper distance. As in the radial case, the phase of relativistic neutrinos for the nonradial motion has been obtained without resort to the weak field approximation. Assuming that the gravitational field is weak enough and the source and the detector are at a sufficiently large distance from the massive object, the phase is reduced to a simpler form as given in Eq. (59). Finally, we have considered the gravitational lensing of neutrinos, i.e., the quantum interference when neutrinos propagate through different paths, and have derived the flavor-changing probability $\mathcal{P}\left(\nu_{e} \rightarrow \nu_{\mu}\right)$ as given in Eq. (62).

Even though the measurement of the gravitational effects on the propagation and oscillations of neutrinos is not feasible at present, we think that the understanding of these behaviors themselves is of interest.

Note added. After the completion of this paper, we became aware of the paper by Cardall and Fuller [14], which discussed a similar subject and obtained the results for the radial motion similar to ours.

\section{ACKNOWLEDGMENTS}

N.F. gratefully acknowledges financial support from the Istituto Nazionale di Fisica Nucleare, Italy. 
[1] B. Pontecorvo, Zh. Eksp. Teor. Fiz. 33, 549 (1957) [Sov. Phys. JETP 6, 429 (1958)]; Z. Maki, M. Nakagawa, and S. Sakata, Prog. Theor. Phys. 28, 870 (1962); B. Pontecorvo, Zh. Eksp. Teor. Fiz. 53, 1717 (1969) [ Sov. Phys. JETP 26, 989 (1967)].

[2] C. Giunti, C. W. Kim, and U. W. Lee, Phys. Rev. D 44, 3635 (1991).

[3] For other approaches, see, for example, J. Wudka, Mod. Phys. Lett. A 6, 3291 (1991); D. Piriz, M. Roy, and J. Wudka, Phys. Rev. D 54, 1587 (1996).

[4] M. Gasperini, Phys. Rev. D 39, 3606 (1989); A. Halprin and C. N. Leung, Phys. Rev. Lett. 67, 1833 (1991).

[5] D. V. Ahluwalia and C. Burgard, Gen. Relativ. Gravit. 28, 1161 (1996); D. V. Ahluwalia and C. Burgard, Report No. gr-qc/9606031 (unpublished).
[6] Y. Grossman and H. J. Lipkin, Phys. Rev. D 55, 2760 (1997).

[7] T. Bhattacharya, S. Habib, and E. Mottola, Report No. gr-qc/9605074 (unpublished).

[8] N. Fornengo, C. Giunti, C.W. Kim, and J. Song (in progress).

[9] C.W. Kim and A. Pevsner, Neutrinos in Physics and Astrophysics (Hardwood Academic, Chur, Switzerland, 1993).

[10] L. Stodolsky, Gen. Relativ. Gravit. 11, 391 (1979).

[11] S. Bilenky and B. Pontecorvo, Phys. Rep. 41, 225 (1978).

[12] C.W. Misner, K.S. Thorne, and J.A. Wheeler, Gravitation (W.H. Freeman and Company, San Francisco, 1973)

[13] S. Weinberg, Gravitation and Cosmology (Wiley, New York, 1972).

[14] C. Y. Cardall and G. M. Fuller, Phys. Rev. D 55, 7960 (1997). 